The knowledgebase project involves:

- content creation (collating information, graphics and other resources);

- an editorial process to ensure consistency and alignment with agreed policies;

- development of the web pages (programming, asset preparation for graphics and videos, layout/design of each page).

The project has a number of goals: People often don't read policy documents, and if they do, they are unlikely to remember the detail over time. At the moment, finding actionable information on policies and procedures is not straightforward and can be time consuming (and therefore wasteful).

Procedures on the inpatient unit in particular have evolved organically over time and there is inconsistency in the knowledge that individuals retain about both routine and less common procedures and medical conditions.

The knowledgebase can improve patient safety by providing interactive tools to simplify drug calculations and drug compatibility checks. It can also provide simple guides on how to set up and maintain equipment.

Paper documentation, charts, guides etc. are stored in a myriad of locations at present; pigeon holes, box folders, cupboards and drawers. The knowledgebase would provide a central, easy to navigate repository of documentation, including printable resources such as charts.

Each section of the knowledgebase will distil and elucidate the most important aspects of individual policy documents and provide access to the full texts should an individual need to read more.

The knowledgebase will complement and reinforce both online and face-to-face training by providing a permanent information resource for clinical staff.

\section{P-70 INTRODUCING AND EMBEDDING EQUALITY, DIVERSITY AND INCLUSION IN CHAS: A COLLABORATIVE APPROACH}

Morven MacLean, Sarah Secombes, Will McLean. Children's Hospices Across Scotland (CHAS), Edinburgh, UK

\subsection{6/bmjspcare-2019-HUKNC.93}

As the national provider of children's hospice care in Scotland, CHAS has a duty to ensure its services are open, accessible and culturally competent for all. In the past, departments worked in silos when it came to equality, diversity and inclusion (EDI), resulting in small successes that were often short-lived. At times, wider organisational barriers prevented some really positive work from being sustainable.

EDI is a way of thinking which will not only widen our clinical reach, but drive improvement and innovation through broader perspectives and diverse thinking. To achieve success, it was critical that we worked collaboratively across departments, integrating and embedding EDI in all that we do.

Fundraising, volunteering and care colleagues came together under the leadership of the Director of Transformation and Innovation to develop an EDI strategy that underpins the CHAS strategy. This new EDI strategy was championed by the Senior Leadership Team and we are now in the process of developing action plans across departments to realise change across CHAS. EDI spans every area of our work, from facilities to nursing and from donor care to recruitment.

Taking an organisational approach demonstrates our commitment to achieving lasting change. Championed by staff who are passionate about EDI, the case for change was clearly presented and understood by senior leaders and the Board.

Change will not happen overnight. However, we now have a clear framework to drive the organisation forward. We know that taking EDI seriously will develop CHAS in so many ways. We want to harness diversity to drive performance and reach as many families in Scotland as possible and are keen to showcase to other organisations that embedding EDI in a meaningful way can be both practical and achievable.

\section{P-71 EQUALITY AND DIVERSITY AT ASHGATE HOSPICECARE}

Sarah Parnacott, Paul Whitehouse. Ashgate Hospicecare, Chesterfield, UK

\subsection{6/bmjspcare-2019-HUKNC.94}

Ashgate Hospicecare identified that it needed to become more diverse to enable it to engage fully with the local community. It launched 'Let's Talk Inclusion' events. This was a key strategic priority for three main reasons. One, to ensure that its services were equitable and available for the whole of the community of North Derbyshire, two, to enable it to have access to key health care professionals and volunteers and be seen as a leading employer in the area and thirdly to have the support from the whole community for its retail and fundraising endeavours.

Links were made with the local Iman and the Muslim community in Chesterfield. They provided education on the care of the Muslim patient at end of life. We were invited to the mosque to be with the community as they broke fast during Ramadan. Fifteen members of staff accepted the invitation. Members of the Muslim community are now volunteers at the hospice. Members of other faith groups are speaking to the hospice.

In February 2019 we had a speaker from the LGBTQ+ community talk about the history of gay rights and related civil rights movements. We launched our own Ashgate Hospicecare rainbow badge and launched our first LGBTQ+ and allies group. Staff and volunteers work together to plan for Pride Chesterfield and other ways to ensure that the hospice engages and meets the needs of those that identify from this part of our community.

We have engaged with charities that support those with sight loss and hearing impediments and those that work with the travelling community and homeless.

Human Rights training has excited hospice staff and there is a planned rollout of this across the hospice.

'Let's Talk Inclusion' has gathered momentum within the hospice and within short months tangible evidence is emerging of the difference this is making for our patients, relatives, staff and volunteers. 